\title{
HEALTHCARE-ASSOCIATED INFECTIONS AT INTENSIVE CARE UNIT OF DEPARTMENT OF ANESTHESIOLOGY AND INTENSIVE MEDICINE AT MARTIN FACULTY HOSPITAL
}

\author{
Lukáš Muajda', Mroslav Šulaj', Henrieta Hudečková', Slavka Litvová̉, J anka Buchencováa \\ 'Department of Public Health, J essenius Faculty of Medicine, Comenius University, Martin, Slovakia \\ 2Department of Anesthesiology and Intensive Medicine, J essenius Faculty of Medicine CU and Martin Faculty Hospital, Martin, Slovakia \\ ${ }^{3}$ Department of Epidemiology, Regional Public Health Authority, Trenčín, Slovakia
}

\begin{abstract}
SUMMARY
We analyzed the occurrence of healthcare-associated infections (HAI) at intensive care unit of the Department of Anesthesiology and Intensive Medicine of Martin Faculty Hospital in 2008. We performed a retrospective-prospective observation according the protocol of European HELICS (Hospital in Europe Link for Infection Control and Surveillance) system. We found $11 \mathrm{HAI}(4.45 \%)$ which on average prolonged the length of hospitalization by 6 days. The most frequent localisation of HAI was the respiratory tract. By our own observation we found the same number of HAI cases as it had been reported officially but the observed and reported cases do not match. The surveillance system HELICS uses definitions slightly different from those used in Slovakia. The severity of health status of a patient at admission influences the risk of HAI. We suggest a continuing collaboration on HELICS system with further involvement of all departments of Martin Faculty Hospital and creation of a hospital infection control team. We also suggestan improvement in testing for etiologic agents of $\mathrm{HAl}$ and an introduction of methods of molecular epidemiology in diagnostics, as well as quantification of costs related to occurrence of $\mathrm{HAl}$ and to assess an implication of automated monitoring system in HAl surveillance.
\end{abstract}

Key words: infection, nosocomial, intensive care, healthcare-associated

Adtress forcomespondance: L. Murajda, Department of Public Health, J FM CU, Sklabinská 26, 03601 Martin, Slovakia.

E-mail: murajda@ gmail.com

\section{INTRODUCTION}

Healthcare-associated infections (HAI) have always accompanied the provision of healthcare. Long ago they seemed inevitable (1). Currently, HAI are a common cause of morbidity and mortality in hospitalized patients worldwide. They create an economic disbalance in making use of resources for the primary and secondary healthcare. They cut short finances from the already low healthcare budget due to causes that are preventable. The experts call for a more profound study of the matter $(2,3,4)$.

Healthcare is offered to a population with increasing susceptibility to infections (ageing, immunodeficience) and severe underlying diseases (e.g. diabetes). The diagnostic and therapeutical procedures create a higher risk of infectious complications (intensive care, invasive interventions, transplantations, etc.). Therefore the importance of HAI is growing and healthcare systems invest more time and resources to solve this problem (5).

According to data from the Epidemiological Information System (EPIS) of the Slovak Republic, in past 10 years (1999-2008) the Department of Anesthesiology and Intensive Medicine (DAIM) reports the highest occurrence of HAI (3.01\%) in Martin Faculty Hospital (MFH). There were 71 cases of HAI in 2,362 patients hospitalized at this department. However, in scientific literature the occurrence at similar departments is usually higher. Podstatová (6) says that the assessed incidence in hospitals is
5-10\% and at departments of ICU type it may be even $20 \%$. That is why we decided to perform our study at DAIM.

\section{MATERIAL}

We observed all patients hospitalized at DAIM in 2008, who stayed at the department for 2 or more days.

\section{METHODS}

We did a retrospective-prospective observation of HAI occurrence at the DAIM according the protocol "Surveillance of Nosocomial Infections in Intensive Care Units" (7) of the European HELICS system (Hospital in Europe Link for Infection Control and Surveillance). The HELICS system is a product of the IPSE (Improving Patient Safety in Europe) project executed in collaboration with the World Health Organisation (WHO), the European Society of Clinical Microbiology (ESCMID) and surveillance networks in European countries, funded by the European Commission Directorate General for Health and Consumer Protection (DG SANCO).

An infection is considered as acquired at ICU if it starts after 48 hours from admission to a ward. Only patients staying more 


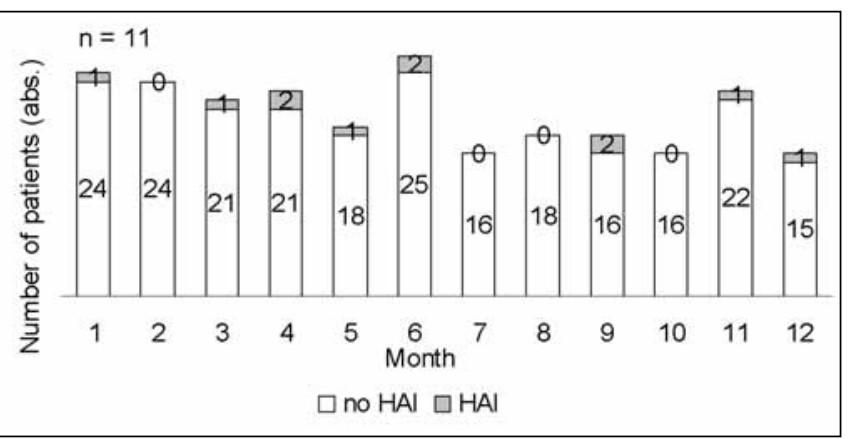

Fig. 1. Healthcare-associated infections (HAI) incidence at Department of anesthesiology and intensive medicine (DAIM) during months in 2008.

than two calendar days enter the study because patients hospitalized less than 48 hours cannot acquire the HAI per definitionem.

There are four main HAI types in HELICS system: bloodstream infection, pneumonia, central venous catheter-related infection and urinary tract infection. Beside that it is possible to observe other infections, grouped as "others".

We created a database from the data acquired and with these data we performed an analysis of the HAI occurrence at DAIM in 2008 using HELICSwin V1.03 program and Microsoft Excel 2003. We analyzed the data according a standard set of HELICS variables: age, gender, type of admission, patient origin, infection site, SAPS II score (Simplified Acute Physiology Score II), trauma before admission, infection date, microorganism and antimicrobial treatment. The SAPS II score is used in emergency medicine to evaluate the risk of dying during hospitalization. It expresses the severity of health status of a patient at admission to a ward.

We counted the length of stay (LOS) of the patients with HAI in 2008 according to our data. We compared the average LOS in patients with HAI and in observed patients without HAI.

We used the t-test and $\chi^{2}$ test for statistical evaluation.

\section{RESULTS}

During the study period (year 2008) there were 247 patients hospitalized at the DAIM. Among all patients hospitalized at DAIM in 2008, there were 125 patients (51\%) hospitalized for 2 days or more. We encountered 11 HAI which matched the HELICS definitions. It is $4.45 \%$ from all patients hospitalized at DAIM in 2008 (Fig. 1).

Among patients with HAI there were 7 men (64\%) and 4 women (36\%). The HAI incidence in women was 13.1 HAI/1,000 patient-days, in men $12.28 \mathrm{HAI} / 1,000$ patient-days. The difference between men and women was not statistically significant ( $p>0.05)$.

Most of patients researched in the study - 98 (78\%) - came from an ICU at MFH or other hospital. Other four patients were transferred from a different ward than ICU (3\%). There were 22 patients (18\%) admitted from community. One patient was admitted from long term care/ nursing home (1\%). The average LOS in observed patients was 7 days (min. 3, max. 31, SD $\pm 5,42$ ).

The HAI incidence in observed 125 patients staying for 2 days or longer was $12.57 \mathrm{HAI} / 1,000$ patient-days.

The highest HAI incidence was in the age group of 40-59 years (18.8 HAI/1,000 patient-days) and 75-79 years (13.6 HAI/1,000

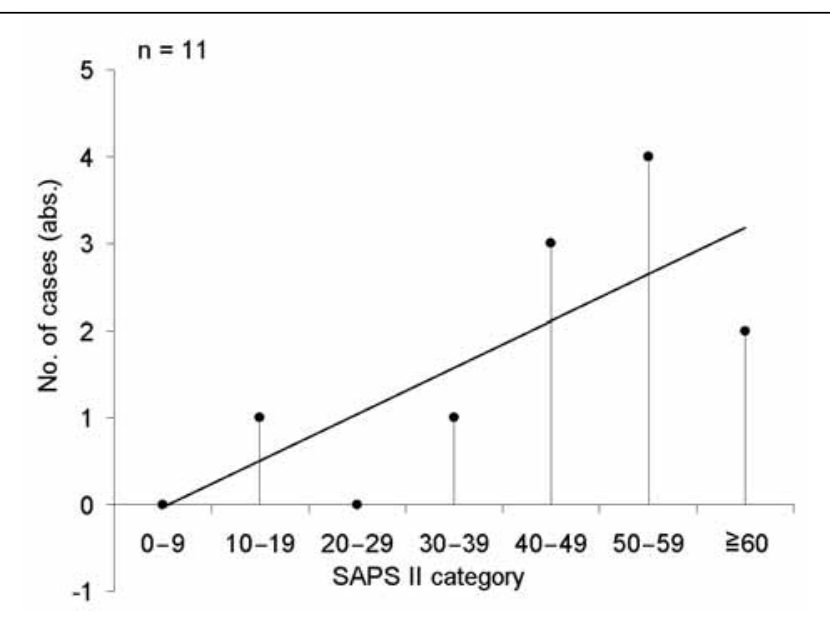

Fig. 2. Healthcare-associated infections (HAl) incidence according the Simplified acute physiology score II (SAPS II) categories among observed patients at Department of anesthesiology and intensive medicine (DAIM) in 2008.

patient-days). However, in both groups it was not statistically significant, compared to other age groups ( $\mathrm{p}>0.05)$.

We found 8 cases of healthcare-associated pneumonia (9.1 HAI/1,000 patient-days). We also found 1 case of bloodstream infection, 1 case of urinary tract infection and 1 case of other type of HAI, incidence of each three being the same: $1.1 \mathrm{HAI} / 1,000$ patient-days.

We saw a higher HAI incidence in patients with higher SAPS II score (Fig. 2).

According to type of admission (medical, scheduled/unscheduled surgical) there were most HAI cases in patients admitted for medical type of admission (7 cases). There were 4 cases in patients with unscheduled surgical admission. There were no patients admitted for a scheduled surgical treatment.

According to patient origin (ICU, other than ICU, community, long term care/nursing home) we found most cases in patients coming from an ICU at MFH or other hospital (8 cases). There were $3 \mathrm{HAI}$ cases in patients from community. However, we did not prove a statistical significance of the higher incidence in patients coming from ICUs ( $\mathrm{p}>0.05)$.

The HAI incidence in patients admitted after a blunt or penetrating traumatic injury was $29.3 \mathrm{HAI} / 1,000$ patient-days (7 cases). The incidence in patients without an injury was 6.3 HAI/1,000 patient-days (4 cases).

There was a statistically significant $(\mathrm{p}=0.035, \mathrm{p}<0.05)$ difference between an average $\mathrm{LOS}$ in patients with $\mathrm{HAI}(\varnothing=12.6$ days, $\mathrm{SD} \pm 8.3)$ and without HAI ( $\varnothing=6.5$ days, $\mathrm{SD} \pm 4.8)$. Patients with HAI were hospitalized on average 6 days longer than patients without HAI.

There were different microorganisms cultivated from samples from patients with HAI. In two cases it was the Pseudomonas aeruginosa and in other two Escherichia coli. In other cases it was Klebsiella sp., Acinetobacter sp., Stenotrophomonas maltophilia, Enterococcus faecium, Enterobacter cloaceae, Citrobacter freundii and Gram-negative sticks or combination of them. Two of the found bacteria had an extended spectrum of $\beta$-lactamases (ESBL). In two cases the causative agent was not identified.

In two cases there were administered antibiotics to which the microbe was clearly resistant (antibiogram). 


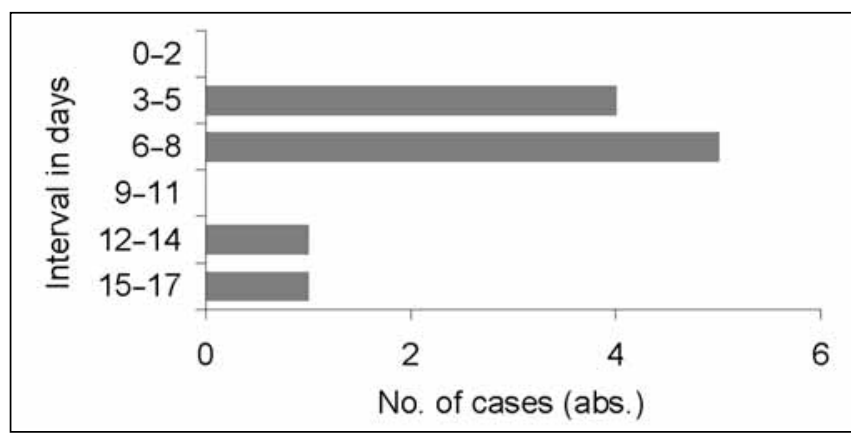

Fig. 3. Interval from admission to hospital to healthcare-associated infections (HAl) occurrence, Department of anesthesiology and intensive medicine (DAIM), 2008.

\section{DISCUSSION}

The higher HAI occurrence at DAIM compared to other departments in MFH reflects the exigence and invasivity of procedures performed. This ward also collaborates on volunteer HAI reporting in European HELICS surveillance system, which indirectly increases the awareness about the burden of HAI and about the importance of their monitoring. However, it is also true that in the past two years the departments of anesthesiology and intensive medicine in Slovakia reported the highest incidence (6.04\%) among all types of departments - the average overall incidence was $0.49 \%$ (8).

The ICU of the DAIM has a 6-bed capacity (one bed in isolation room). According the HELICS protocol only the patients staying longer than 48 hours and more enter the study. Therefore we observed only this group of patients (125 patients). Even if the patients staying less than 48 hours were not included, they still present a considerable part of the patients at DAIM and it is necessary to take into account the risk of spreading some pathogens which they pose when transferred to other wards.

Most of the patients (78\%) were transferred from other ICU in MFH or other hospital. The high percentage is characteristic for this ward. It expresses the severity of health status of the patients admitted and highlights the risk of transmitting the HAI from original wards.

Healthcare-associated pneumonia was the most common HAI. Ventilator-associated pneumonia is the most important HAI in patients at ICU wards (9). They are the second most frequent HAI and they have the highest letality. In intensive care, their incidence can reach $65 \%$ (3).

The HAI definitions according HELICS are very exact and for our conditions even strict. An important part of the HAI definition is a culture proof and repeated X-ray. When assessing the patient records we repetitively found a probable HAI according to clinical health status of a patient but we could not define it as a HAI because it did not meet some of necessary criteria. Otherwise the number of HAI could have been higher (pneumonia in particular).

We think it may be suitable to adjust our national HAI definitions to those used in other EU countries to facilitate benchmarking. Realisation of this goal exceeds our authorization, of course.

The increased HAI incidence in patients with higher value of the SAPS II score confirms the importance of severe basic health status as a risk factor for HAI.

The higher HAI incidence in patients after preceding trauma in comparison with the incidence in patients without HAI suggests that trauma is a risk factor for HAI. Trauma usually damages the integrity of surface of skin or mucosa, it weakens the patient and often requires an invasive therapy.

The interval between admission and HAI occurrence was 3-17 days ( $\varnothing=7.2$ days; $S D \pm 4.2$ ). In the early phase of hospitalization there occurred 4 cases of HAI, in the later phase 7 cases. Šrámová et al. (10) say that in the early phase of hospitalization (under the 4th day) the HAI are caused mostly by endogenous strains of patients themselves. These strains (Streptococcus pneumoniae, Hemophilus influenzae, $\beta$-hemolytical streptococci, some enterobacteria) tend to be sensitive to antibiotics. In the later phase (after the 5th day) there appear exogenous HAI caused mostly by multiresistant bacterial strains. Thus, we may assume that in observed patients some more exogenous HAI did occur.

When comparing causative agents of HAI in Martin - Pseudomonas aeruginosa, Escherichia coli, Klebsiella sp., Acinetobacter, Stenotrophomonas maltophilia, Enterococcus faecium, Enterobacter cloaceae, Citrobacter freundii a Gram-negative bacilli - and microbes causing HAI in Slovakia most frequently - Staphylococcus sp., Klebsiella sp., Pseudomonas sp., E.coli, Proteus sp. and Acinetobacter (8) - we see that Klebsiella sp., Pseudomonas sp., E. coli and Acinetobacter are frequent in both. It is interesting that in HAI cases in our study we did not identify Staphylococcus sp. as a causative agent in any of the cases. However, in literature Staphylococcus $s p$. is considered the major cause of HAI generally (11).

We evaluated the length of stay (LOS) of patients hospitalized at DAIM in 2008. We confirmed that in these patients HAI on average increase the LOS by 6 days. Number of HAI cases found is lower because also the number of hospitalized patients is lower as well. Despite of that the number of patient days lost due to HAI is relatively high - approximately 66 excessive patient days from the total number of 875 patient days which is $7.5 \%$ of all patient days.

The excessive LOS in relation to HAI represents a loss of patient days. It can be seen as a loss of payments from health insurance institutions, because the income of hospital and its wards depends on number of completed hospitalizations in the Slovak Republic. Calculation of such loss is complicated under our conditions because the health insurance institutions put limits on the number of hospitalizations.

That is why the constant payments for every completed hospitalization, HAI represent a higher loss for the healthcare facility than for health insurance institutions (12). One needs to consider the loss of payments from health insurance institutions, treatment cost, diagnostics, running of a ward, wages for personnel, suffering, deterioration of quality of life, longer working incapability and possible lawsuits.

For a successful infection control at DAIM it is necessary to improve surveillance in the whole Faculty Hospital in Martin. Currently we consider as a key target an improvement in HAI reporting. This goal is generally a problem of all hospitals in Slovakia (13) and its fulfilment is not easy.

In scientific articles we encounter success of hospital programs of infection control. A good example is the American National System for Surveillance of Nosocomial Infections (NNIS). As shows the report Monitoring Hospital-Acquired Infections to Promote Patient Safety (14), NNIS is a volunteer hospital reporting system for HAI, created in '70s in the USA for HAI monitoring 
and for coordination of preventive efforts of infection control practitioners. According this report the hospitals should have at least one infection control specialist for every 250 hospital beds. In case of the MFH this would mean approximately 3-4 work positions.

A practical handbook of HAI prevention (9), published by the World Health Organization, does not only speak about the need of infection control doctor, but also about a hospital infection control service, about a team of different workers within infection control.

In many countries they use a model based on results of the SENIC study which defines a necessity of one hospital hygienist for each 250 beds and one epidemiologist or microbiologist for each 1,000 beds (15). Van den Broek et al. (16) discussed the number of people in the infection control team. Their work comes out from the actual situation in Netherlands. They suggest to increase the standard of SENIC study to one hospital hygienist for each 178 beds and one microbiologist for each 806 hospital beds. They suggest to count these according to the number of admitted patients, which means one hospital hygienist on 5,000 admissions and one microbiologist on 25,000 admissions. In MFH it would be a group of approximately 5 hospital hygienists and one microbiologist.

In developed countries the trend leads to still greater implication of electronic information systems. Bouam et al. (17) describe a comparison of an intranet automatized system of surveillance of HAI with direct observation and reporting done by medical doctors in one French faculty hospital. According to their results, an intranet automatized system is more exact and less time-consuming.

There is an electronic database system in MFH, primarily appointed for analysis of the data on antibiotic therapy (RAL) which is used for internal needs of MFH (18). To some extent it gives an indirect information on HAI occurrence and could be implemented in a HAI surveillance program.

According Beaglehole et al. (19) the reporting system of HAI cases is the most important part of surveillance. Gastmeier et al. (20) state that in order to succeed in preventive activities and surveillance, independently from a system of surveillance used, the conditio sine qua non is a close collaboration with employees at all wards of a hospital.

\section{CONCLUSION}

We consider it beneficial to continue with the collaboration on European system of surveillance HELICS at DAIM and to involve in its activity also the other departments at MFH. We consider it useful to elaborate an internal guideline for the hospital on HAI surveillance and to observe costs related to HAI incidence at different wards and the hospital as a whole.

For a successful solution of the problems with HAI, it is needed to create an infection control team, composed from experts on hospital hygiene, epidemiology and microbiology. Such a group would have, according to known studies, 3-4 or even 6 members. Based on successful examples from abroad, we suggest also the use of electronic information system within the HAI surveillance.

A better HAI surveillance in MFH could decrease significantly the number of HAI and also the suffering of patients. It would eliminate a big part of the financial burden posed by HAI and release considerable finances for quality improvement of the healthcare offered.

\section{REFERENCES}

1. Möse JR. Hygiene as an academic discipline at Austrian universities: a survey on the occasion of the 120th anniversary of the Institute of hygiene in Graz. Cent Eur J Public Health. 2005 Mar;13(1):6-10.

2. Gastmeier P. Nosocomial infection surveillance and control policies. Curr Opin Infect Dis. 2004 Aug;17(4):295-301.

3. Mad'ar R, Podstatová R, Rehořová J. Prevention of nosocomial infections in clinical practice. 1st ed. Prague: Grada; 2006. (In Czech.)

4. Kolářová M, Šrámová H, Príkazský V, Míčková E, Jirouš J, Kancelová Z, et al. Prevalence study of nosocomial infections at surgical departments of faculty hospitals in the Czech Republic. Hygiena. 2006;51(3-4):81-6. (In Czech.)

5. Exner M, Peters G, Engelhart S, Mielke M, Nassauer A. 1974-2004: the Commission for Hospital Hygiene has been working for 30 years. From the "old" to the "new" guideline. Bundesgesundheitsblatt Gesundheitsforschung Gesundheitsschutz. 2004 Apr;47(4):313-22. (In German.)

6. Podstatová H. Basic epidemiology and hygiene. 1st ed. Prague: Galén; 2009. (In Czech.)

7. HELICS-ICU working group. Surveillance of nosocomial infections in intensive care units Protocol. Version 6.1. September 2004. IPH/ EPI reports D/2004/2505/48 [Internet]. Brussels: Scientific Institute of Public Health; 2004 [cited 2010 May 24]. Available from: http://helics. univ-lyon1.fr/protocols/icu_protocol.pdf.

8. Lokša P. Nosocomial infections are also in the Slovak Republic a constant danger. Med Pract. 2009;5:4-5. (In Slovak.)

9. Ducel G, Fabry J, Nicolle L, editors. Prevention of hospital-acquired infections: a practical guide. 2nd ed [Internet]. Geneva: World Health Organization; 2002 [cited 2010 May 24]. Available from: http://www. who.int/csr/resources/publications/drugresist/whocdscsreph200212.pdf.

10. Šrámová H, et al. Nosocomial infections II. Prague: Maxdorf; 2001. (In Czech.)

11. Satorres SE, Alcaráz LE. Prevalence of icaA and icaD genes in Staphylococcus aureus and Staphylococcus epidermidis strains isolated from patients and hospital staff. Cent Eur J Public Health. 2007 Jun;15(2):87-90.

12. Dietrich ES, Demmler M, Schulgen G, Fekec K, Mast O, Pelz K, et al. Nosocomial pneumonia: a cost-of-illness analysis. Infection. 2002 Apr;30(2):61-7.

13. Annual report on activities of regional public health authorities in the Slovak Republic 2008 [Internet]. Bratislava: Public Health Authority of the Slovak Republic; 2009 [cited 2010 May 24]. Available from: http:// slovanet.sk/uvzsr/docs/vs/vyrocna_sprava_SR_08.pdf. (In Slovak.).

14. Centers for Disease Control and Prevention (CDC). Monitoring hospitalacquired infections to promote patient safety--United States, 1990-1999. MMWR Morb Mortal Wkly Rep. 2000 Mar 3;49(8):149-53. Erratum in: MMWR Morb Mortal Wkly Rep 2000 Mar 10;49(9):189-90.

15. Haley RW, Culver DH, White JW, Morgan WM, Emori TG, Munn VP, et al. The efficacy of infection surveillance and control programs in preventing nosocomial infections in US hospitals. Am J Epidemiol. 1985 Feb;121(2):182-205.

16. Van den Broek PJ, Kluytmans JA, Ummels LC, Voss A, VandenbrouckeGrauls CM. How many infection control staff do we need in hospitals? J Hosp Infect. 2007 Feb;65(2):108-11.

17. Bouam S, Girou E, Brun-Buisson C, Karadimas H, Lepage E. An intranetbased automated system for the surveillance of nosocomial infections: prospective validation compared with physicians' self-reports. Infect Control Hosp Epidemiol. 2003 Jan;24(1):51-5.

18. Krkoška D, et al. Antibiotics and nosocomial infections. In: Kavcová E, Szárazová M, editors. Health promotion, prevention and hygiene in theory and practice - III. Martin: Jessenius Faculty of Medicine, Comenius University; 2004. p. 248-59. (In Slovak.)

19. Beaglehole R, Bonita R, Kjellstrom T. Basic epidemiology. 4th ed. Geneva: World Health Organization; 2000.

20. Gastmeier P, Sohr D, Just HM, Nassauer A, Daschner F, Rüden H. How to survey nosocomial infections. Infect Control Hosp Epidemiol. 2000 Jun;21(6):366-70.

Received November 4, 2009 Accepted in revised May 25, 2010 